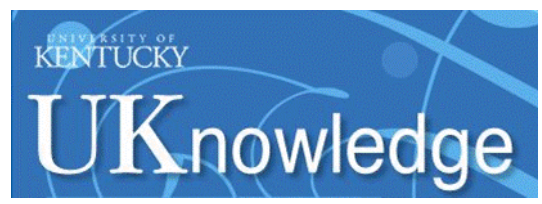

University of Kentucky

UKnowledge

$1-2002$

\title{
Retrospective Analysis of Youth Evaluated for Suicide Attempt or Suicidal Ideation in an Emergency Room Setting
}

Julie Hagedorn

University of Kentucky

Hatim A. Omar

University of Kentucky, hatim.omar@uky.edu

Follow this and additional works at: https://uknowledge.uky.edu/pediatrics_facpub

Part of the Emergency Medicine Commons, Mental and Social Health Commons, and the Pediatrics

Commons

Right click to open a feedback form in a new tab to let us know how this document benefits you.

\section{Repository Citation}

Hagedorn, Julie and Omar, Hatim A., "Retrospective Analysis of Youth Evaluated for Suicide Attempt or Suicidal Ideation in an Emergency Room Setting" (2002). Pediatrics Faculty Publications. 86.

https://uknowledge.uky.edu/pediatrics_facpub/86

This Article is brought to you for free and open access by the Pediatrics at UKnowledge. It has been accepted for inclusion in Pediatrics Faculty Publications by an authorized administrator of UKnowledge. For more information, please contact UKnowledge@lsv.uky.edu. 


\section{Retrospective Analysis of Youth Evaluated for Suicide Attempt or Suicidal Ideation in an Emergency Room Setting}

\section{Digital Object Identifier (DOI)}

https://doi.org/10.1515/IJAMH.2002.14.1.55

\section{Notes/Citation Information}

Published in International Journal of Adolescent Medicine and Health, v. 14, no. 1, p. 55-60.

(C) Freund Publishing House Ltd.

The copyright holder has granted permission for posting the article here.

Reprinted as a book chapter in Suicidal Behavior in Adolescence: An International Perspective. Joav Merrick, \& Gil Zalsman, (Eds.). p. 191-196. 


\section{Retrospective analysis of youth evaluated for suicide attempt or suicidal ideation in an emergency room setting} Julie Hagedom, MD and Hatim Omar, MD.

Section of Adolescent Medicine, Department of Pediatrics, University of Kentucky, Lexington. United States of America

Abstract: Suicide is the third leading cause of death in adolescents and a major contributor to morbidity in this age group. The objective of this study was to look at the demographics, major stressors and factors leading to attempting suicide as well as the methods of attempted suicide in adolescents admitted to two hospitals in a medium size city. Medical records were reviewed of adolescents admitted to two area hospitals for attempted suicide between 7/1/97-12/31/99. Coroner's data on completed suicide were also reviewed. In the study period a total of 287 persons aged 21 years or under were admitted for attempted suicide. Mean age was 16.9 years (range $7-21$ ). $53.4 \%$ of the total were females and 46.6 were males with the majority of the total being Caucasians (75.6\%). Interpersonal conflicts were the most common stressors preceding the attempt; fight with parents $20 \%$, end of a relationship $12 \%$, fight with a significant other $8 \%$. Financial difficulties were the culprit in $10 \%$ of the cases. Abuse was not clearly recorded in $64 \%$ of cases. In cases where documentation was clear, $60 \%$ reported sexual and $67 \%$ physical abuse. Nearly half of the patients had a prior psychiatric diagnosis with prior suicide attempt and depression being most common at 27 and $18 \%$ respectively. Overdose was the most common method utilized. There were 20 completed adolescent suicides in the area with firearms as the method used in all of them. It is concluded that suicide continues to be a major problem in adolescents. Access to guns may be a detrimental factor in completing suicide. Health care providers may help identify those at risk by routinely screening all adolescents for depression and suicide. :

Keywords: Adolescence, adolescent suicide, adolescent violence, depression, adolescent mortality, mental health, risk-taking behavior, United States

Correspondence: Hatim Omar, MD, Associate Professor, Director of Adolescent Medicine \& Young Parent programs, J422, Kentucky Clinic, University of Kentucky, Lexington, KY 40536-0284, United States. Tel: 859-323-6426 ext. 307, Fax.859-257-7706, E-mail: haomar2@pop.uky.edu

Submitted: January 11, 2002. Revised: January 18, 2002. Accepted: January 19, 2002.

\section{INTRODUCTION}

Numerous studies have indicated that suicide is a serious problem among youth. Several studies have determined a $9 \%$ suicide attempt rate in high school students (1-3). A study by Dubow estimated that $7 \%$ of high school students attempt suicide and $36 \%$ contemplate it (4). A twenty-one year longitudinal study by Fergusson reported suicidal ideation in $28.8 \%$ of youth and suicide attempts in $7.5 \%$ by age 21 . (5). Similarly, the Youth Risk Behavior Surveillance System survey of a nationally representative sample of high school students found that $20.5 \%$ had contemplated and $7.7 \%$ had attempted suicide in the preceding twelve months (6).

Psychological autopsies of youth successfully completing suicide have identified numerous factors more significantly 
associated with suicide completers than with controls. These factors include nonintact family of origin, less frequent and satisfying communication with parents, history of mood disorder in mother, history of legal problems in father, family history of suicidal behavior, recent discipline (especially school suspension and juvenile court appearance), recent breakup with girlfriend or boyfriend, recent separation of parents, lack of employment or school attendance, grade failure (7), and presence of DSM III diagnosis (8), especially mood disorder, anxiety disorder, and disruptive disorders.

According to prior studies, predictors of suicide attempts include parental depression, poor family functioning (9), large number of parental changes, poor attachment to parents, exposure to sexual abuse (5), depression, hopelessness (10), anxiety disorder, and substance abuse (11). Primary reasons reported for suicide attempts, when given a list to choose from, include to die, relief from state of mind, escape from a situation, and to make others understand how desperate one feels (12).

Negron, in a 1997 study (13), found fighting with parents or others, and school problems, to be the most common stressors preceding suicide attempt or ideation. Few other studies have examined such stressors. In an effort to further understand youth suicide and to aid in early identification of those at risk, this study retrospectively examined preceding stressors, preexisting psychiatric diagnoses, methods/plans of suicide attemptideation, prior abuse, and various demographic data.

\section{METHODS}

Medical records of adolescents 21 years of age or under admitted to two area hospital emergency rooms between July 1997 and December 1999 for attempted suicide were reviewed. Data regarding method or plan of suicide attempt or ideation, stressors preceding the attempt/ideation, history of abuse, preexisting psychiatric diagnoses, and various demographic data as recorded in psychiatric consult notes and in emergency physician notes were collected and analyzed. A total of 287 cases were identified and reviewed (see Table 1), with an age range of 7.21 years (mean of 16.9 years).

\section{RESULTS}

A total of 287 patients were admitted for attempted suicide between 7/1/97-12/31/99. Caucasians were the majority at $75.6 \%$ and females were slightly more than males (see Table 1). History of abuse was not clearly identified in $184(64 \%)$ cases, however in 103 cases where abuse was documented the incidence of sexual abuse was $60 \%$ and physical abuse $67 \%$. In addition, $50 \%$ of patients had a prior psychiatric diagnosis with prior suicide and depression topping the list at 27 and $18 \%$ respectively.

Fifty percent of these patients were discharged with outpatient follow-up, $47 \%$ were admitted to a psychiatric inpatient unit, and $3 \%$ were admitted to other units (ICU, Surgery, Jail etc.). During the same period, 20 adolescents completed suicide in the area and in all cases of completed suicide, firearms were utilized.

The most common stressors leading to attempting suicide are listed in Table 2 with interpersonal conflicts with parents, ending a relationship and fight with a significant other leading the list. Overdose was the most commonly used method of suicide (see Table 3). Interestingly, $3 \%$ of patients used intentional car crashes as a method of attempting suicide. This is significant, $0.7 \%$ because car crashes are usually listed under accidents, which may alter the overall national data on causes of morbidity and mortality.

\section{DISCUSSION}

This study found a fairly equal gender distribution of youth who contemplate or 
Table 1. Demographic characteristics of adolescents admitted for attempted suicide.

\begin{tabular}{ll}
\hline Mean Age & 16.9 years \\
Age Range & $7-21$ years \\
Females & $53.4 \%$ \\
Males & $46.6 \%$ \\
Caucasians . & $75.6 \%$ \\
African American & $21.4 \%$ \\
Hispanic & $1.5 \%$ \\
Asian & $0.8 \%$ \\
Others & \\
\hline
\end{tabular}

Table 2. Most common stressors reported as leading to suicide.

Fight with Parent

End of a relationship

Fight with a significant other

Financial problems

Recent move, social isolation

Legal problems

Family Problems

Academic problems

Substance abuse

Homosexuality

Recent abuse

Other stressors
$20 \%$

$12 \%$

$8 \%$

$10 \%$

$7 \%$

$6 \%$

$6 \%$

$5 \%$

$4 \%$

$3 \%$

$4 \%$

$15 \%$

Table 3. Methods utilized in the suicide attempts.

\begin{tabular}{ll}
\hline Overdose & $32 \%$ \\
Cut or stab by self & $15 \%$ \\
Intentional car accident & $3 \%$ \\
Hanging & $2 \%$ \\
Threats with pointing guns, Jumping, etc. & $32 \%$ \\
All other methods & $18 \%$ \\
\hline
\end{tabular}


attempt suicide, suggesting that primary care physicians and specialists alike need to worry about suicide in both genders. This differs from the male dominance found in suicide completers (14). Data obtained from the Fayette County Coroner's Office in Kentucky indicated that $17(85 \%)$ of 20 suicides were committed by males (Penn, personal communication). Data from the same source showed a higher rate of suicide in the later teenage years ( $70 \%$ were sixteen to eighteen years of age). This is similar to results of this study, suggesting that late adolescence can be a very difficult time, perhaps due to multiple changes and the challenges faced when moving toward adulthood and independence. Addressing these issues may help identify those at risk for suicide or even help prevent some suicidal ideation and attempts.

Other risk factors that physicians need to address, according to this study, include history of prior suicide attempt, depression, substance abuse, and disruptive disorders (attention deficit hyperactivity disorder, conduct disorder, oppositional defiant disorder). These findings are in agreement with prior studies as mentioned above $(4,5,7,11-13)$. In contrast, the number of youth with no prior psychiatric diagnosis equaled those with a prior diagnosis of depression in this study, suggesting that clinicians should not assume that only those with a history of problems are at risk.

Examination of life stressors may be one way to identify those at risk for suicidal ideation or attempt, who have never been diagnosed with a psychiatric disorder. The most common stressor found in this study was fighting with a parent. This is somewhat surprising, although concordant with prior studies $(9,11)$, as adolescents often seem more concerned with their relationships with others than with their relationships with their parents as they attempt to gain independence and separate from their parents. Perhaps the difficulty of these tasks makes the child-parent relationship all the more important so that when it deteriorates, youth feel distressed enough to contemplate or attempt suicide. Clinicians can help alleviate this distress by routinely questioning children and adolescents about parental and other relationship problems many of the other stressors revolved around interpersonal relationships, as prior studies also found -- and by intervening to improve these relationships.

Problematic interpersonal relationships were not the only important stressors identified in this study. As in prior studies $(4,10,13)$, financial, academic, and legal difficulties were also shown to be common stressors prior to suicide attempt or ideation. Physicians ought to ascertain the extent of these problems in their patients to help identify those at risk for suicide. They also should ask about physical and sexual abuse. As indicated in this study, abuse is a common stressor, but clinicians often do not ask about it.

In addition, physicians need to consider the possible lethality in overdose of medicines that they prescribe to potentially suicidal patients, as overdose was the most common method of suicide attempted and contemplated. Over-the-counter medications were most commonly used. Thus, patients should be counseled on the lethality of these medications when recommended as well. These results differ from those in suicide completers. According to Fayette County, Kentucky coroner's data from 1993-2000, gunshot wound (75\%) and hanging $(15 \%)$ were more common than overdose $(5 \%)$ (Penn, personal communication). Thus, it is important to assess the availability of guns to potentially suicidal patients. In addition, this difference suggests that those who complete suicide may have a greater tendency than those who don't choose more lethal means of attempt.

Finally, outpatient follow-up was the most common disposition found in those 
evaluated. It is unclear why more children were not hospitalized after suicide attempt. or ideation. Perhaps many of the children showed remorse for their actions, or there was indication that the attempts were: impulsive and without intent to die. Whatever the case, the findings indicate that suicidal ideation and attempts do not always require hospitalization, and are problems that clinicians may have to deal with more and more in the outpatient setting.

Due to the retrospective design, this study has certain limitations. As histories were obtained by numerous physicians, there were differences in the quality of data gathered, as evidenced by the poor documentation of abuse. Use of structured interviews would have ensured the gathering of more uniform data. In addition, due to the retrospective design, accuracy of prior diagnosis could not be ensured as prior diagnosis was merely recorded by the physician as reported by the patient or as mentioned in earlier medical records. Also, the data analysis system used may not have identified all patients evaluated for suicidal ideation or attempt. Medical records of patients with more serious attempts may have been coded for stab wound, gunshot wound, motor vehicle accident, and other injuries rather than for suicide attempt. The Local source of data in a medium size city environment may be different than rural or large urban areas.

\section{CONCLUSION}

Suicide continues to be a major problem in adolescents. Access to guns may be a detrimental factor in completing suicide. Health care providers may help identify those at risk by routinely screening all adolescents for depression and suicide.

\section{ACKNOWLEDGEMENT}

This study was presented in part at the $15^{\text {th }}$ annual meeting of the North American Society of Pediatric and Adolescent
Gynecology, Toronto, Canada, May 18-20, 2001.

\section{REFERENCES}

1. Centers for Disease Control. Attempted suicide among high school students-United States, 1990. MMWR 1990; 40:633-5. Harkavy F, Jill M, Asnis GM, Boeck M, DiFiore J. Presence of specific suicidal behaviors in a high school sample. Am J Psychiatr 1987;144:1203-6.

2. Smith K, Crawford S. Suicidal behavior among "normal" high school students. Suicide Life Threatening Behavior 1986;16:313-25.

3. Dubow E, Kausch D, Blum M, Reed J, Bush E. Correlates of suicidal ideation and attempts in a community sample of junior high and high school students. J Clin Child Psychol 1989;18:158-66.

4. Fergusson DM, Woodward LJ, Horwood LJ. Risk factors and life processes associated with the onset of suicidal behavior during adolescence and early adulthood. Psychol Med 2000;30:23-39.

5. Kann $\mathrm{L}$, et al. Youth risk behavior surveillance - United States, 1997. J School Health 1998; 68:355-69.

6. Gould MS, Fisher P, Parides M, Flory M, Shaffer D. Psychological risk factors of child and adolescent completed suicide. Arch General Psychiatr 1996; 53:1155-62.

7. Shaffer D, Gould MS, Fisher $P$, Trautman P, Moreau D, Kleinman M, Flory M. Psychiatric diagnosis in child and adolescent suicide. Arch General Psychiatr 1996; 53: 339-48.

8. Garber J, Little S, Hilsman Ruth, Weaver KR. Family predictors of suicidal symptoms in young adolescents. J Adolesc 1998;21:445-57.

9. Overholser JC, Frejheit SR, DiFilippo JM (1987), Emotional distress and substance abuse as risk factors for 
suicide. Can J Psychiatr 1997;42:402-8.

10. Gould MS, King R, Greenwald S, Fisher P, Schwab-Stone M, Kramer R, Flisher AJ, Goodman S, Canino G, Shaffer D. Psychopathology associated with suicidal ideation and attempts among children and adolescents. J Am Acad Child Adolesc Psychiatr 1998; 37: 91523.

11. Boergers J, Spirito A, Donaldson D. Reasons for adolescent suicide attempts: associations with psycho logical functioning. J Am Acad Child
Adolesc Psychiatr 1998; 37: 1287-93.

12. Negron R, Placentini J, Graae F, Davies $M$, Shaffer D. Microanalysis of adolescent suicide attempters and ideators during the acute suicidal episode. $J$ Am Acad Child Adolesc Psychiatr 1997;36:1512-9.

13. Kaplan H, Sadock B. Suicide, violence, and other psychiatric emergencies $\left(2^{\mathbf{d}}\right.$ Ed). In: Susan Gay, ed. Pocket Handbook of Clinical Psychiatry. Baltimore, MD: Williams and Wilkints, 1996: 206.

\section{BITS 'N PIECES}

Prevalence and trends in overweight and obesity in three cross sectional studies of British children, 1974-94

Objectives: To report trends in overweight and obesity, defined by new internationally agreed cut-off points, in children in the United Kingdom. Design: Three independent cross sectional surveys.

Setting: Primary schools in England and Scotland.

Participants: 10414 boys and 9737 girls in England and 5385 boys and 5219 girls in Scotland aged 4 to 11 years.

Main outcome measures: Prevalence and change in prevalence of overweight and obesity, as defined by the international obesity task force, in 1974, 1984, and 1994, for each sex and country.

Results: Little change was found in the prevalence of overweight or obesity from 1974 to 1984 . From 1984 to 1994 overweight increased from $5.4 \%$ to $9.0 \%$ in English boys (increase $3.6 \%, 95 \%$ confidence interval $2.3 \%$ to $5.0 \%$ ) and from $6.4 \%$ to $10.0 \%$ in Scottish boys (3.6\%, $1.9 \%$ to $5.4 \%$ ). Values for girls were $9.3 \%$ to $13.5 \%(4.1 \%, 2.4 \%$ to $5.9 \%)$ and $10.4 \%$ to $15.8 \%(5.4 \%, 3.2 \%$ to $7.6 \%)$, respectively. The prevalence of obesity increased correspondingly, reaching $1.7 \%$ (English boys), 2.1\% (Scottish boys), 2.6\% (English girls), and 3.2\% (Scottish girls).

Conclusion: These results form a base from which trends can be monitored. The rising trends are likely to be reflected in increases in adult obesity and associated morbidity.

Chinn S, Rona RJ. BMJ 2001;322:24-6, 